\title{
Chapter 18 \\ Renewing Climate Planning Locally in the Tropics: Conclusions
}

\author{
Maurizio Tiepolo, Alessandro Pezzoli and Vieri Tarchiani
}

\begin{abstract}
In the Tropics, a significant increase in the number of cities provided with climate plans by 2020, as announced in the 11th Sustainable development goal of the United Nations, requires an unprecedented effort. To achieve it, we have to simplify the planning process and improve the quality of the plans. The aim of this book was to collect methods and experiences to inspire the simplification of the planning process and increase the quality of climate planning. We focused attention on the three critical phases of the planning process: analysis, decision making in planning, climate measures. Sixteen case studies from Ethiopia, Haiti, Malawi, Mexico, Niger, Senegal, Tanzania and Thailand cover automatic weather stations in remote areas, rainfall estimation gridded datasets, open data for vulnerability index to climate change, early warning systems, quality of climate plans index, multi-risk local assessment, flooding risk evaluation method, backcasting, spatial dimension in disaster risk reduction and resilience, gasification stoves, index-based insurance and vulnerability risk credit. After indicating the possible analyses, 19 recommendations were supplied to the United Nations SDGs monitoring system, the national weather services and those responsible for natural risks, to the Development banks, Official development aid and the research institutions.
\end{abstract}

Keywords Climate trends - Climate measures - Climate planning • Decision making $\cdot$ SDG $\cdot$ Vulnerability tracking $\cdot$ Tropics

M. Tiepolo is author of 18.1, 18.2.2 and 18.3 sections. A. Pezzoli is author of 18.2.1 and 18.2.3 sections. V. Tarchiani is author of 18.2 and 18.4 sections.

M. Tiepolo $(\varangle) \cdot$ A. Pezzoli

DIST, Politecnico and University of Turin, Viale Mattioli 39, 10125 Turin, Italy

e-mail: maurizio.tiepolo@polito.it

A. Pezzoli

e-mail: alessandro.pezzoli@polito.it

V. Tarchiani

National Research Council-Institute of BioMeteorology (IBIMET),

Via Giovanni Caproni 8, 50145 Florence, Italy

e-mail: v.tarchiani@ibimet.cnr.it 


\subsection{From the State of Climate Planning to the Book Approach}

This book contributes to "substantially increase the number of human settlements adopting and implementing integrated policies and plans towards resource efficiency, mitigation and adaptation to climate change, resilience to disasters, and disaster risk reduction", in short, to what we have called "climate planning". That announced is one of the targets of the 11th Sustainable development goal of the United Nations, "Make cities inclusive, safe, resilient and sustainable" which the signees undertake to achieve by 2020 . We proceeded by 4 steps.

First of all, we decrypted the concept of climate planning expressed in the New urban agenda (2016) and in the Sendai framework for disaster risk reduction (2015) of the United Nations.

Secondly, we identified the category of cities which are expected to prevail in the Tropics in 2020 and 2030 (Chap. 1).

Third, we ascertained the state of climate planning in large and medium-sized cities in the Tropics, identified local access to the key information necessary to climate planning and the most common climate measures (Chaps. 1 and 10).

Four, we compared vision (UN SDGs) and reality (urbanization trends, state of climate planning, key-information access, most frequent climate measures). The comparison highlights three critical points of the process which the 11th SDG aims to put in place.

\subsubsection{New Tools and Measures}

The climate plan proposed by the United Nations is that usually applied in the large cities of OECD and BRICS countries, where stand-alone plans or the acclimatisation of long-term general, comprehensive and master plans prevail. But in 2030, the urban population in the Tropics will be concentrated mainly in cities under 0.3 million pop. (46\%), even if large cities will see the biggest increase (from today's 38-44\%). Medium-size tropical cities mainly use medium-term municipal development plans because they do not have sufficient resources to prepare and run additional tools or because no other plans are required by the national laws. Consequently, the plans that should be produced from now on and the measures that should be implemented to limit climate change and its impacts on human settlements should be different from those that local administrations are currently implementing in application of the single national legislations. This requires a regulatory adjustment that cannot be achieved in the short term. The alternative is to continue producing stand-alone plans that are detached from the local legal system and which have little chance of being appropriated and implemented (becoming an expenditure item in the municipal budget) once external financing ends. 


\subsubsection{Increasing the Production of Plans}

The pace sustained by climatic planning in the last seven years has more than doubled the number of climate plans in the Tropics compared to the previous seven years. Today, just 104 cities in the most advanced tropical countries (OECD and BRICS) still don't have one. But no fewer than 950 cities in tropical DC and LDCs still have no tools to reduce emissions or cope with the impacts of climate change, and half of these are in countries with no climate planning experience. If the production of plans could keep up this pace until 2020, not even half of the large and medium-sized tropical cities would have a climate plan. And this can certainly not be considered a substantial increase in climate planning.

\subsubsection{Increasing the Quality of the Plans}

Our survey of 338 tropical cities revealed an even lower quality of the plans, which is inevitably translated into a poor impact of the measures envisaged. Two percent of climate plans estimate the potential impact of the planned measures, only $6 \%$ of the plans characterizes the local climate change and only one out of five climate plans specifying the quantity of measures to be taken. This uncertainty creates further inaccuracies: source of funding (indicated by one plan out of four) and the cost of measures (indicated by $42 \%$ of the plans only). The analysis and decision making tools are therefore two weaknesses of the examined plans. Knowledge of climate change and its impacts is often lacking. And when knowledge exists it is acquired in a way that would radically renewed. For example, the preliminary analyses of planning (hazard, hazard-prone zones, vulnerability) are occasional, rarely become vulnerability tracking and are almost never locally accessible. This aspect is not considered by the SDGs monitoring system. We see this as a limit: what is not monitored cannot be accomplished.

We have reached the conclusion that, in order to achieve the above-mentioned target a radical renewal of local climate planning is necessary.

Producing new plans, more than in the past and of better quality, requires the simplification and acceleration of certain steps of the current planning process. It is with this intention that the book uses sixteen case studies to tackle three key-steps of the planning process:

(1) Analysis for local climate planning

(2) Decision making tools for local planning

(3) Innovation in climate measures 


\subsection{Lessons Learned from the Book}

\subsubsection{Analysis for Local Planning}

Analysis for local climate planning includes characterisation/probability of hazard in the contexts exposed to climate change, in the medium-long term, which is the horizon of climate plans (from 5 to about 20 years). In these cases, the analysis is called up to characterise the climate, taking into account the needs for agricultural and livestock production, protection of key infrastructures and, more generally, of human settlements. In greater detail, we have seen (Chap. 2) that it is necessary to ensure a technical capacity in maintaining and operating AWS-automatic weather stations safety equipment. AWS are particularly important for flood monitoring, which is often carried out in remote watersheds compared to the built-up areas on the plain, which are frequently large and medium-sized cities.

We then have to consider the new threats due to climate change: intense rainfall and dry spells also in drought treated countries (Chap. 3). Importance of tailored climate analysis (at local scale) with daily resolution (not seasonal trends like IPCC) superimposing natural hazard likelihood with other indicators.

For farming activities (e.g.: urban and peri-urban) it is useful to have high resolution daily precipitation gridded datasets: probability of unfavourable conditions for crops (according to critical periods in growing stages) and favourable trends (late end of the rainy season) which can, however, trigger conflicts with herders (Chap. 4).

The analysis also implicates the ascertainment of vulnerability to climate change, which can be necessary on different scales: regional, using the municipality as a minimum unit of analysis, or restricted to parts of the smallest administrative jurisdiction. Depending on the contexts, these are occasional assessments, as in the case of the Ethiopian districts (Chap. 5), or tracking, where open data exist, as in Haiti (Chap. 6). The open access of the single indicators (e.g.: that on flood exposure) guaranteed by a national service, would allow every local authority to directly access information on exposure and vulnerability relating to its jurisdiction on a scale sufficient to its local planning needs, accelerating the local analysis process and cutting spending, which could be addressed towards measures for adaptation. The diagnosis for planning includes the Early Warning System. This device also belongs to the measures but is considered here to ascertain that the hazard prone areas are effectively covered by EWS. Also the device for extreme precipitation alerts in Malawi uses open source information (Chap. 7).

When monitoring cropping season, it is necessary to plan things with caution, paying attention to translating information products into services that should be simple but effective for farmers, establishing a communication and collaboration between scientific institutions, national technical services and rural communities for an effective dissemination and adoption of the advice (Chap. 8). Climate services for farmers should be integrated into a concerted and participatory strategy (including content and format of advice, communication channel, partnership with 
other local stakeholders and training), which enables final users to adopt scientific innovation in ways that are socially acceptable and environmentally sound. The approach needs to pay special attention to gender: in many cases, women are the backbone of the farming community, however they are disadvantaged in accessing climate and weather information as well as training programs.

\subsubsection{Decision Making Tools for Climate Planning}

The huge effort that has gone into climate planning in the last seven years has been carried out largely by the large and medium sized cities of the member countries of the OECD and BRICS, but much less than by those of the DC and LDCs (Chap. 10). The quality of planning measured with the QCP Index composed of 10 indicators remains low.

Climate planning is expected to spread, but it should also rise in quality, something which can be appreciated with the QCPI-Quality of Climate Planning Index (Chap. 10). This requires an improvement in methods, integrating the concept of risk for example. The MLA-Multirisk Local Assessment (Chap. 11) presents various advantages compared to the Community Risk Assessment. For example, it identifies the potential impacts of climate change and those of the risk reduction methods. The cost of potential damages compared with risk reduction costs allows the identification of the benefits of the single measures, also in the frequent cases of communities exposed to multiple risks. Lastly, the method combines technical knowledge and local knowledge. The FREM-Flooding Risk Reduction Method integrates in participated methods for flood risk management, a robust analysis of local condition in a context where local capacities on climate and prediction are absent (Chap. 12).

The use of forecasting in the case of measures to increase access to water in Dar es Salaam can be integrated with the identification of shared community objectives (backcasting). The first method is effective in establishing the technical limits of an intervention and can be helped by the definition of possible scenarios with the second method (Chap. 13).

These methods however require integration into local procedures. An example is supplied by disaster risk reduction and resilience in the case of La Paz, Mexico. The method proposed allows an assessment of urban resilience with a spatial dimension (Chap. 14).

\subsubsection{Innovation in Climate Measures}

As far as the measures are concerned, it is a matter of innovating the range available, focusing on those more capable of mitigating emissions and risk, and 
then financing them. Two measures are almost completely absent from the 346 plans for tropical cities considered by our survey.

The first regards the use of alternative fuels to wood and coke for cooking. The "three stone" is still the most frequently used stove in many large tropical cities. The effects of this device on emissions of $\mathrm{CO}_{2}$ and on deforestation (reduction of carbon sinks) around cities are clear. It alters the plant coverage, increasing run-off and soil erosion, which aggravate floods. The use of crop residues to produce pellets and special gasification stoves is an answer to deforestation and to environmental alteration, which could impact on climate change mitigation (Chap. 15). Its use in an urban context would allow the limitation of the incessant process of deforestation which has exceeded a radius of $100 \mathrm{~km}$ around Niamey, the capital city of Niger, affecting most of the watersheds responsible for the disastrous floods in 2010, 2012 and 2016.

The second measure is the index-based insurance to share risk in the case of adverse weather events to which farming is exposed (Chap. 16), rarely provided for in local climate plans. This, however, is a measure that has experienced an impasse but which has potential if accompanied by measures for risk reduction.

Lastly, the question of financing the measures. The VRCs-Vulnerability Reduction Credits system (Chap. 17) presented and tested in the case of Niger can allow the channelling of funds towards plans which are likely to have the biggest impact.

\subsection{Areas for Future Research}

Climate plans are a way of mainstreaming climate change mitigation and risk reduction measures. However, they run the risk of remaining unimplemented and of not changing the conducts of people and key players. This is why it is necessary to act in other ways at the same time.

Key infrastructures (production and distribution of energy, roads, railways, ports, dams or important urban services, from education to health) could have a considerable impact on the reduction of emissions and the reduction of risk. The possible impact of infrastructures on climate change and, vice versa, of climate change on the construction and exercise of such infrastructures, if considered right from the concept of the project stage, can be substantially reduced. The critical entrance point are the Development banks. The request for specific climatic requirements in the proposals of financing submitted to the Development banks has an impact of the entire growth machine: designers, surveyors, construction firms and maintenance firms, suppliers of machinery, construction material, local administration and sectorial administration of the Government.

A second sector is that of technical standards for the construction of acclimatized infrastructures.

Attention should be extended to these two sectors in the future. 


\subsection{Recommendations}

Nineteen recommendations to different stakeholders are triggered by the book.

\section{United Nations SDGs monitoring system}

1. Consider the percentage of large and medium-sized cities with climate plans as an indicator for the monitoring of the 11th SDG.

2. Consider the quality of the climate plans as an indicator for the monitoring of the 11th SDG (QCPI).

3. Use a database on cities and climate planning, similar to that created for this book, and update it.

\section{National Hydro-meteo Services}

4. Integrate the different sources of climate data. In LDCs and specially in remote areas, the observation networks are few and far between. Climate analysis on a local scale (municipal) can be practiced successful, integrating data observed through the network of automatic weather stations and rainfall estimation gridded datasets like TRMM, CHIRPS, etc.

5. Publish the data on floods so that local planning can be based on the analysis of past events also in semi-arid countries, where floods are becoming a high risk, both in urban and rural areas. Developing flood reporting systems based on geo-referenced data and on the longest possible timescale.

\section{National Services Responsible for Natural Risks}

6. Switch from vulnerability assessment to vulnerability tracking, possibly through open data with a reduced number of indicators and make access to information open.

7. Make all the information useful to local climate planning freely available, from climate data to the areas exposed to flood, landslide, sea level rise and drought, to farmland, the prices of farm products on the markets, forestry coverage, victims and damage after disaster, through to school enrolment by gender.

8. Consider the capacity to react to vulnerability.

\section{Federal/State Authorities Responsible for Local Development Planning Guidelines}

9. Increase the number of climate measures in the municipal development plans.

10. Prepare lists of recommended measures, depending on the potential impact.

11. Make climate plans more detailed, both in analysis and in the measures, which should be structural and non-structural (e.g.: index-based insurance, climate services, etc.).

12. Introduce into municipal development plans the assessment of the residual risk as a tool to make decisions regarding the measures that can have the biggest impacts on the reduction of risk. 


\section{Research Institutions}

13. Switch from monitoring the climate plans to tracking plan implementation.

14. Ascertain the state of climate planning in towns (under 0.1 million pop.).

15. Extend the survey on the other entry points of climate action: infrastructure projects and construction standards.

16. Ascertain the impact of the most important measures to reduce risk, in detail.

\section{Official Development Aid}

17. Assess adaptation measures in projects in terms of VRC-credits.

\section{Insurance sector}

18. Relaunch index-based insurance.

\section{Development Banks}

19. Finance projects for transition to the gasification stove fuelled by pellets, in urban and rural areas.

Open Access This chapter is licensed under the terms of the Creative Commons Attribution 4.0 International License (http://creativecommons.org/licenses/by/4.0/), which permits use, sharing, adaptation, distribution and reproduction in any medium or format, as long as you give appropriate credit to the original author(s) and the source, provide a link to the Creative Commons license and indicate if changes were made.

The images or other third party material in this book are included in the book's Creative Commons license, unless indicated otherwise in a credit line to the material. If material is not included in the book's Creative Commons license and your intended use is not permitted by statutory regulation or exceeds the permitted use, you will need to obtain permission directly from the copyright holder.

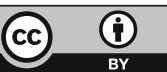

